\title{
Performance Evaluation of Azimuth Offset Method for Mitigating the Ionospheric Effect on SAR Interferometry
}

\author{
Wu Zhu, Wen-Ting Zhang, Yu-Fang He, and Wei Qu \\ School of Geology Engineering and Geomatics, Chang'an University, Xi'an, Shaanxi, China \\ Correspondence should be addressed to Wu Zhu; zhuwu@chd.edu.cn
}

Received 27 April 2017; Revised 17 July 2017; Accepted 24 July 2017; Published 18 September 2017

Academic Editor: Saro Lee

Copyright ( $2017 \mathrm{Wu}$ Zhu et al. This is an open access article distributed under the Creative Commons Attribution License, which permits unrestricted use, distribution, and reproduction in any medium, provided the original work is properly cited.

\begin{abstract}
Synthetic aperture radar (SAR) signals interact with the ionosphere layer when they propagate through the atmosphere, leading to the phase delay error for SAR interferometry (InSAR). To mitigate this error for SAR interferometry, azimuth offset method is proposed. However, the performance of it has not been fully investigated. In this situation, this study makes a comprehensive performance analysis of azimuth offset method through processing the simulated and real SAR data. The experimental result indicates that this method can effectively mitigate the ionospheric phase delay error, where the standard deviation of phase difference after correction ( $2.6 \mathrm{rad}$.) decreased by almost 2 times, compared to those before correction (5.3 rad.) for the real SAR data. However, it is also found that the method is affected by the random noise, which may induce the improper estimation of integral constants and consequently affect the ionospheric correction. Moreover, the severe deformation signals in the interferogram may lead to the estimation error of integral constants and scaling factor. Therefore, it should mask out the deformation signals when using the azimuth offsets method to correct the ionospheric error. This study may provide useful information when using azimuth offset method to mitigate the ionospheric phase delay error on InSAR.
\end{abstract}

\section{Introduction}

The phase difference of two synthetic aperture radar (SAR) images acquired for the same scene but at different sensor positions offers the possibility of constructing the topography and detecting the displacement of the surface [1]. Based on this concept, the interferometric SAR (InSAR) technology was developed and has been widely used to investigate the ground displacement associated with earthquakes [2], volcanoes [3], landslides [4], subsidence [5], and other geological processes [6] as well as the atmospheric studies [7]. However, this technology may be affected by the ionospheric phase delay due to the different ionospheric conditions for two interferometric SAR images [8]. Ionosphere layer is one part of the upper atmosphere comprising portions of the mesosphere, thermosphere, and exosphere. Generally, ionosphere varies with the activity levels of the Sun, time, geographical position, and so on. showing the inhomogeneous and periodical variation nature. When SAR signals propagate through the atmosphere, they interact with ionosphere layer with the result that additional time delay, phase advance, and polarization changes are produced.

A series of studies on the ionospheric effects on SAR and InSAR have been published since 1960s, including Faraday Rotation (FR) [9, 10], phase advance [11], SAR resolution, and image shift. But, for the SAR interferometry, it has been demonstrated that the most significant effect was the azimuth streak [12]. The azimuth streak was generated when SAR satellites travelled through the spatial gradient ionospheric layer, leading to the SAR Doppler shift and consequent azimuth pixel shift in interferograms [13], as shown in Figure 1. According to the ionosphere-induced azimuth shift theory, an approximate linear relationship between the azimuth shift and ionospheric phase streaks (IPS) was proposed in 2002 [14]. Based on this relationship, IPS can be estimated and then removed from the ionosphere-contaminated interferograms so as to mitigate the ionospheric effect on SAR interferometry. Raucoules and De Michele [15] initially tried to estimate and correct the IPS by using interferometric 


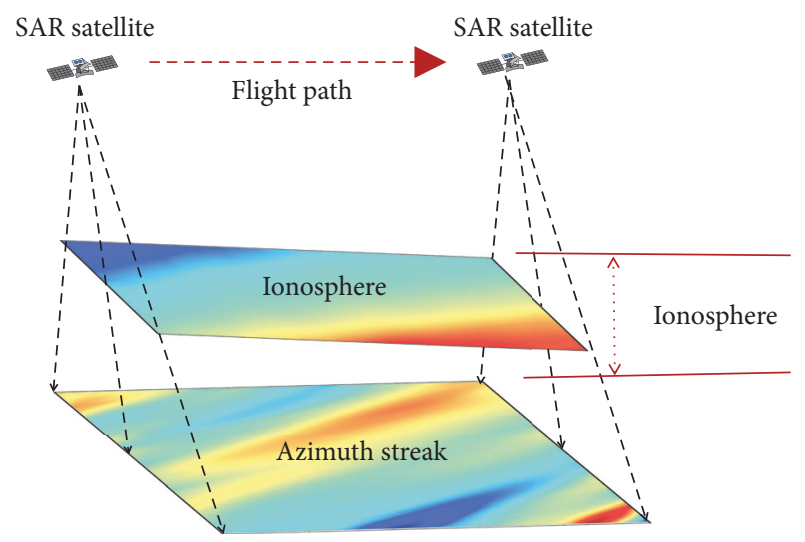

FIGURE 1: The ionosphere-induced azimuth streak.

azimuth offset from the L-band ALOS-1/PALSAR interferograms over the Wenchuan earthquake (China). Their experiment demonstrates the potential for mitigation of the ionospheric error on SAR interferograms by using the azimuth offsets method. Jung et al. [16, 17] and Liu et al. [18] further improved this method by using the Multiple Aperture SAR Interferometry (MAI) technique to estimate the azimuth offset, which is considered to be more efficient and robust in measuring along-track displacements.

Although azimuth offset has been carried out to mitigate the ionospheric phase delay error, the performance of this method has not been fully investigated. Therefore, this study will make a comprehensive performance analysis of azimuth offset method in mitigating the ionospheric phase delay error on SAR interferometry. The structure of this study is arranged as follows: firstly, the methodology is simply introduced in Section 2; after that, data processing with the simulated and real data is described in Section 3; then, result is shown and analyzed in Section 4; finally, discussion and conclusion are drawn in Section 5.

\section{Methodology}

Figure 2 shows the designed flowchart to mitigate ionospheric error on SAR interferometry by using the azimuth offset method. This scheme mainly involves three aspects: estimation of azimuth offset, estimation of IPS, and compensation of IPS on InSAR. The detailed processing methods are described as follows.

2.1. Estimation of Azimuth Offset. The ionospheric disturbance can cause the prominent azimuth shift on InSAR images. This shift can be predicted if the VTEC map on the SAR flight path is available. However, such a VTEC map with short time duration (tens of seconds) and high spatial resolution (a few meters to tens of meters) is usually difficult to be obtained from the current instruments and techniques [19]. On the other hand, the azimuth shift can be conveniently estimated from some complex image registration methods, for example, intensity cross-correlation and MAI technique. Therefore, it is possible to correct the ionospherecontaminated interferogram according to the approximate linear relationship between the azimuth shift and IPS. Based on this analysis, it is essential to estimate the accurate interferometric azimuth offset.

In this study, the Split Beam Interferograms (SBI) technique was employed to estimate the azimuth shifts. The SBI technique was developed by the GAMMA SAR Software Corporation (GAMMA Remote Sensing and Consulting AG, Bern, Switzerland) and uses the same principle with the MAI technique [12]. Basically, the azimuth shift $\Delta x_{\text {shift }}$ based on SBI method is defined as

$$
\Delta x_{\text {shift }}=-\frac{l}{4 \pi n} \phi_{\mathrm{SBI}}
$$

where $n$ is the normalized squint, $l$ is the effective antenna length, and $\phi_{\mathrm{SBI}}$ is the SBI phase. The main processing steps in estimating the azimuth shift by the SBI method are coregistration of Single Look Complex (SLC) images, azimuth spectrum band-pass filtering of SLC images, calculation of forward-looking and backward-looking interferograms, and combination of forward-looking and backward-looking interferograms to produce SBIs, unwrapping filtered SBIs, and transferring unwrapped SBIs to azimuth shifts.

2.2. Estimation of IPS from Azimuth Offset. Once the azimuth shift $\Delta x_{\text {shift }}$ is determined, IPS $\phi_{\text {iono }}$ may be estimated by

$$
\phi_{\text {iono }}=\alpha \int \Delta x_{\text {shift }} d x+c
$$

where $\alpha$ is a system- and geometry-dependent scaling factor and $c$ is the integral constant. Here, the scaling factor $\alpha$ is calculated by a linear fitting of the azimuth shift $\Delta x_{\text {shift }}$ and along-track derivative of the interferometric phase $\dot{\phi}_{\text {azi }}$ :

$$
\dot{\phi}_{\mathrm{azi}}=\alpha \times \Delta x_{\text {shift }}
$$

In order to reduce the distortions caused by other errors, high coherence points are used to determine the scaling factor $\alpha$. As for the integral constant $c$, it is firstly assumed that they have the identical initial value and then compute the initial IPS using (2). The initial IPS might show an obvious gradient in range direction, which is caused by the improper integral constant. In this situation, the integral constant $c$ is updated by averaging the differences between interferometric phase $\phi_{\text {InSAR }}$ and initial IPS. Then the updated IPS is obtained from (2). If the gradient in range direction still remains, this process should be repeated until the gradient disappears.

2.3. Compensation of IPS. Compensation of IPS was carried out by subtracting the IPS $\phi_{\text {iono }}$ from the interferometric phase $\phi_{\text {InSAR }}$ :

$$
\widehat{\phi}_{\text {InSAR }}=\phi_{\text {InSAR }}-\phi_{\text {iono }}
$$

where $\bar{\phi}_{\text {InSAR }}$ is the interferometric phase after compensation of the IPS. After carrying out such a procedure, two polynomial models were applied to remove the residual phase ramp and atmospheric phase with respect to height from 


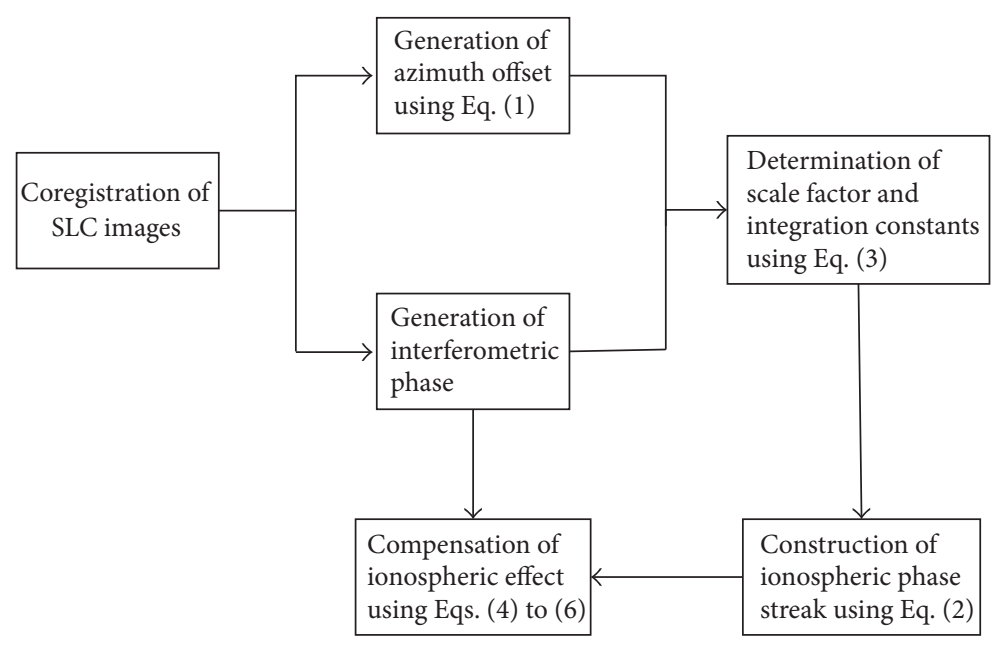

FIGURE 2: Processing flowchart of mitigating of ionospheric phase delay error for SAR interferometry by using azimuth offset method.

the interferometric phase, respectively. Residual phase ramp $\phi_{\text {ramp }}$ was determined by a quadratic phase model:

$$
\phi_{\text {ramp }}=a_{0}+a_{1} x+a_{2} y+a_{3} x y+a_{4} x^{2}+a_{5} y^{2},
$$

where $x$ and $y$ are the pixel locations at range and azimuth direction, respectively, and $a_{0}-a_{5}$ are the model parameters. Atmospheric phase with respect to height $\phi_{\text {atm }}$ is modelled as

$$
\phi_{\mathrm{atm}}=b_{0}+b_{1} h \text {, }
$$

where $h$ is the topographic height and $b_{0}$ and $b_{1}$ are the model parameters. After fitting the two models, we removed them from the ionosphere-corrected interferograms and created a refined interferometric phase.

\section{Data Processing}

3.1. Simulated SAR Data. To assess the performance of azimuth offset method, a test with the simulated data was conducted. In this simulation, the effects of random noise and linear deformation phase on the IPS estimation were concerned. It should be noticed that the other signals were ignored, including topographic errors, atmospheric delay, and orbital error, since they should have had the similar impacts with the deformation signal. Figure 3 shows the simulated ionosphere-induced azimuth offset and corresponding wrapped IPS maps with 300 pixels in range direction and 300 pixels in azimuth direction. The azimuth offset in Figure 3(a) ranges from $-1 \mathrm{~m}$ to $1 \mathrm{~m}$. IPS in Figure $3(\mathrm{~b})$, varying from -9 rad. to $28 \mathrm{rad}$., was calculated from (2) with the assumed scaling factor $\alpha$ and integral constant $c$. Here, the simulated IPS was mainly taken as the true IPS to evaluate the estimated IPS by azimuth offset method in different scenarios.

3.1.1. The Simulated Random Noise. The noise is common in the SAR interferogram, which may be caused by several sources, such as baseline or geometric decorrelation, Doppler centroid differences, volume decorrelation, thermal noise, and temporal terrain decorrelation [8]. Here, the noise with random distribution was simulated. The simulated random noise had the mean value of $30^{\circ}$ and the standard deviation of $10^{\circ}$, which was much more serious than the normal case [20]. Based on the simulated random noise, it was magnified for 1 to 20 times and subsequently added this to the true IPS map (Figure 3(b)). The interferometric phase will become increasingly noisy with the increasing times of the random noise. It is predicted that this noisy interferometric phase may lead to the improper estimation of the scaling factor and integral constants when using the azimuth offset to estimate the IPS.

3.1.2. The Simulated Deformation Phase. In the practical case, the interferometric phase may contain the contributions of deformation phase and IPS, for example, a coseismic interferogram. In this situation, the IPS estimated by the azimuth offset method may be affected by the deformation signals. In order to investigate this influence, the deformation phase was simulated and magnified from 1 to 20 times. Subsequently, the simulated deformation phase added details to the true IPS (Figure 3(b)). After that, the azimuth offset method was used to estimate the IPS.

3.2. Real SAR Data. In order to further test the performance of azimuth offset method in the situation of both deformation and ionosphere signals appearing in the interferograms, two coseismic interferometric pairs over April 13, 2010, for the Yushu, China, 6.9 earthquake were generated in this experiment. Table 1 lists the interferometric parameters, where pair 2 was contaminated by the ionosphere and was corrected by the azimuth offset method. Interferometric pair 1 was used to evaluate the ionospheric correction for pair 2, since it was not affected by the ionosphere.

Figures 4(b) and 4(c), respectively, show the interferometric phase measurement and azimuth offset for pair 2. Figures 4(d) and 4(e), respectively, show the interferometric phase measurement and azimuth offset for pair 1 . 


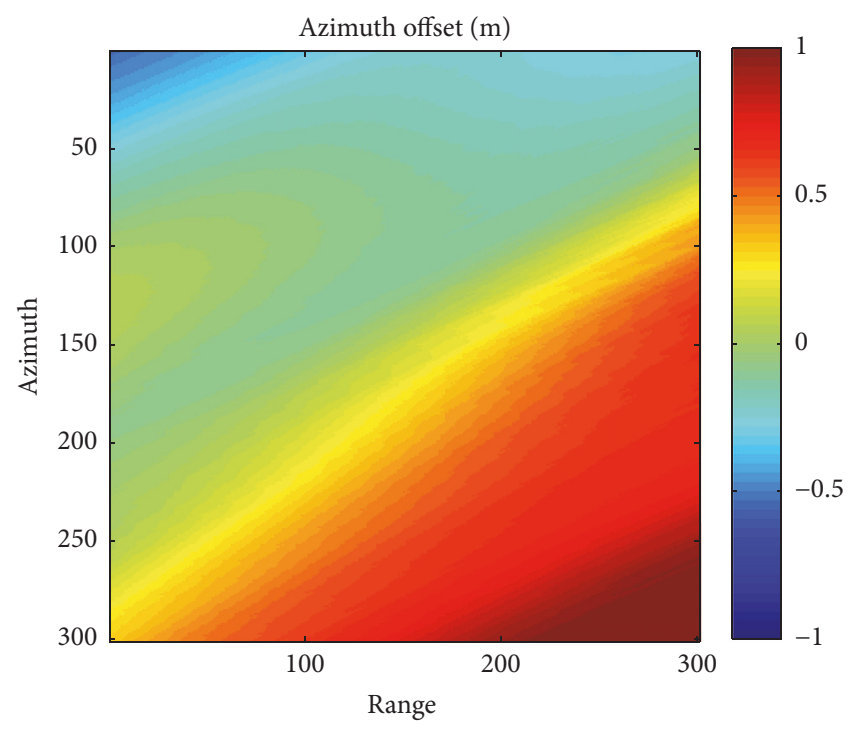

(a)

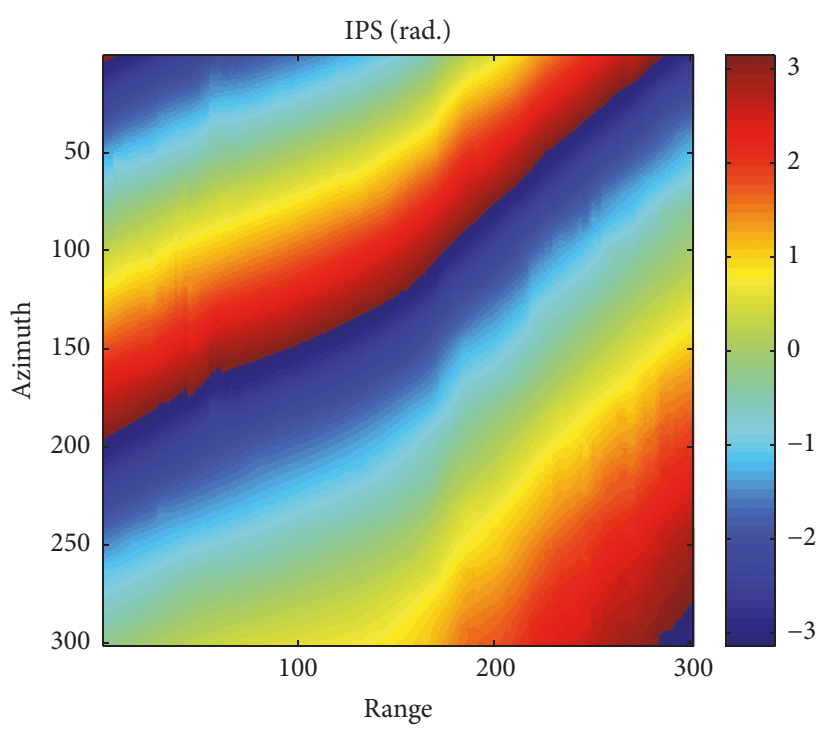

(b)

FIGURE 3: (a) Simulated ionosphere-induced azimuth offset map and (b) corresponding ionospheric phase streak with the assumed scaling factor and integral constant.

TABLE 1: Interferometric parameters for Yushu earthquake area, where $B_{T}$ stands for the temporal baseline and $B_{P}$ stands for perpendicular baseline.

\begin{tabular}{|c|c|c|c|c|c|c|c|c|}
\hline Number & Satellite & Inclination & Master & Slave & Center frequency & Incident angle & $\begin{array}{c}B_{T} \\
\text { (days) }\end{array}$ & $\begin{array}{l}B_{P} \\
(\mathrm{~m})\end{array}$ \\
\hline Pair 1 & ALOS-1 & Ascending & $15 / 01 / 2010$ & $17 / 04 / 2010$ & $1270 \mathrm{MHZ}$ & $38.7^{\circ}$ & 92 & 671 \\
\hline Pair 2 & ALOS-1 & Ascending & $15 / 01 / 2010$ & $18 / 07 / 2010$ & $1270 \mathrm{MHZ}$ & $38.7^{\circ}$ & 184 & 865 \\
\hline
\end{tabular}

It was observed that Figure 4(b) presents the complicated phase patterns. The main contributions might result from the earthquake deformation, water vapor delay, ionospheric delay, topographic error, and orbital noise. In our analysis, it was assumed that deformation, ionosphere, and orbit were the dominant phase. Actually, this is acceptable because Figure 4(b) presents the severe long-wavelength signals in the far field of the earthquake, which is compatible with the spatial characteristics of ionospheric and orbital errors. In the near field of the earthquake, it was dominated by the deformation signal but may be also mixed with the ionospheric and orbital errors. The azimuth offset in Figure 4(c) was estimated by SBI technique. It was found that Figure 4(c) presented the anomalous stripe-shaped fringes, which were mainly introduced by the ionosphere and deformation. Here, the orbital phase contribution was neglected as the azimuth offset estimated by SBI technique was nearly not affected by the orbit [21]. Once the interferometric phase and azimuth offset are produced, the azimuth offset method could be used to estimate the IPS.

As analyzed in the previous section, the calculated azimuth offset map (Figure 4(c)) was dominated by the coseismic deformation signal and ionospheric error. In order to estimate the IPS from azimuth offset, ionospheric contribution should be separated from the azimuth offset map. This effort could be actualized by directional polynomial fitting and linear trend removal [15]. In this case, a thirddegree polynomial was first employed to estimate the azimuth streaks' amplitudes. After that, Figure 4(c) was rotated to align horizontally the streaks and replace each line by its third-degree fit. Finally, the ionosphere-induced azimuth streaks were generated, as shown in Figure 5(b). It can be observed from Figure 5(b) that the most ionosphereinduced azimuth streaks were separated without affecting high-frequency small-scale offsets in the near field of the earthquake. Then, the scaling factor was determined through a linear fitting of the azimuth gradient of interferometric phase (Figure 5(a)) and separated ionosphere-induced azimuth offset (Figure 5(b)) at the selected high coherence points, as shown in Figure 5(c). It should be noticed that the coseismic deformation phase was masked in Figure 5(a) for the sake of the accuracy of the scaling factor. Figures 5(d) and 5(e) display the estimated IPS and the corresponding VTEC map. The visible phase streaks and small-scale ionospheric gradient may be observed from both images. Meanwhile, it was noticed that these streaks showed the similar extending direction with the ionosphere-induced azimuth offset map. In this case, the maximum of phase error due to the ionospheric disturbance was about $-41.8 \mathrm{rad}$., which corresponded to $-0.78 \mathrm{~m}$ deformation error in LOS direction. For the ionosphere, the maximum of VTEC difference between January 15 and July 18, 2010, was 15.66 TECU. 


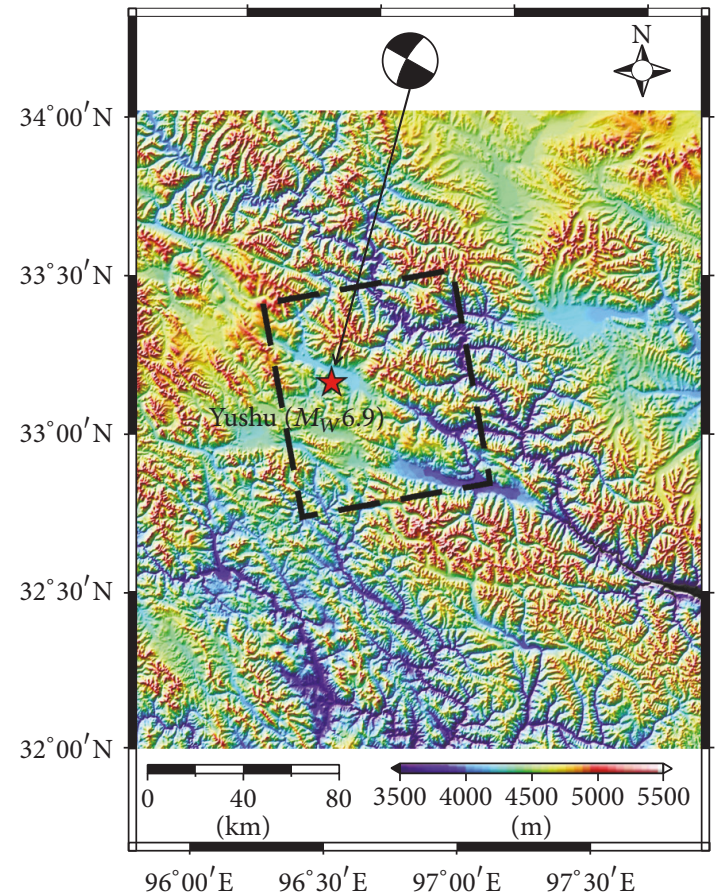

(a)

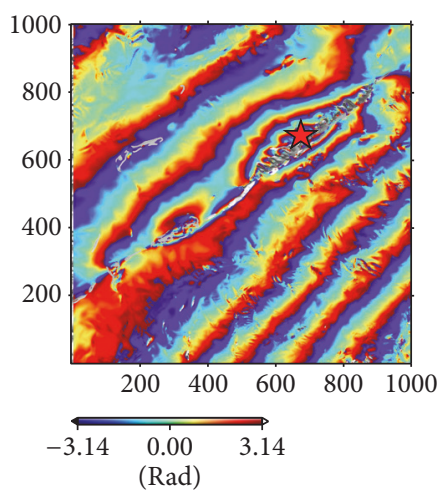

(b)

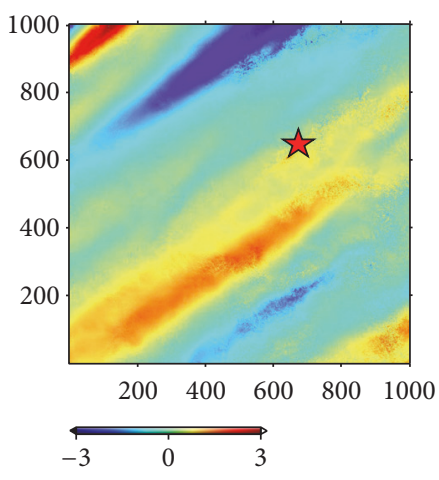

(c)

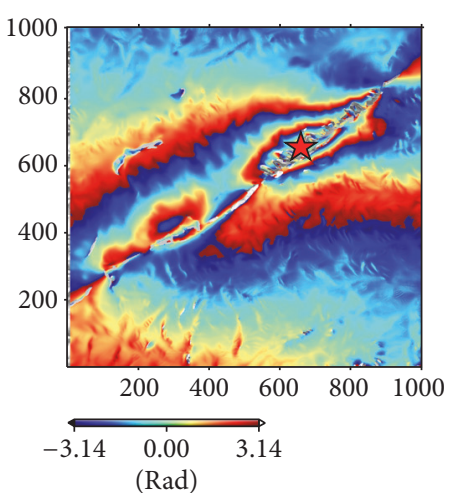

(d)

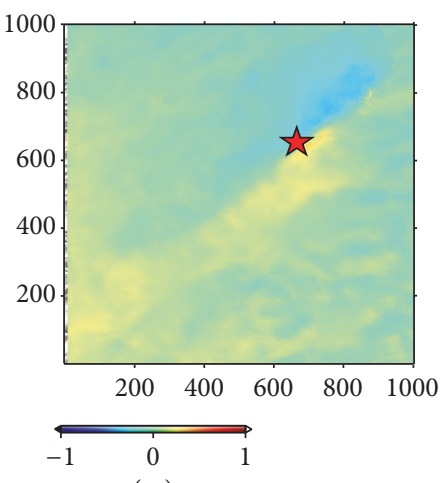

(e)

FIgURE 4: (a) Topographic map of 2010 Yushu earthquake. The black dotted rectangle denotes the spatial coverage of experimental L-band ALOS-1/PALSAR images and red pentagram shows the epicentre positions of the mainshock; (b-c) interferometric phase and azimuth offset between January 15, 2010, and July 18, 2010, respectively; (d-e) interferometric phase and azimuth offset between January 15, 2010, and April 17,2010 , respectively.

\section{Result}

\subsection{Simulated SAR Data}

4.1.1. The Simulated Random Noise. Figure 6 shows the variations of the scaling factor, integral constants, and standard deviation of the bias of the estimated IPS with the magnified random noises from 1 to 20 times. It is found that the scaling factors in Figure 6(a) remain approximately constant with the magnified random noise, suggesting that it is not affected by the random noise. However, the integral constants in Figure 6(b) experience an obvious variation with the magnified random noise. In this case, the integral constants are overestimated at about 0.2 radians for each range pixel when the random noise is magnified twice. Due to the overestimation of integral constants, the bias of the estimated IPS becomes larger and larger with the increasing random noise, as shown in Figure 6(c). From this simulation, it can be concluded that the random noise in the interferogram can induce the improper estimation of integral constants and may consequently produce the discontinuous pinstripe along the range direction in the estimated IPS map.

4.1.2. The Simulated Deformation Phase. Figures 7(a)-7(d) show the simulated deformation phase, variations of the scaling factor, integral constants, and standard deviation of the bias of the estimated IPS with the magnified deformation phase from 1 to 20 times. It can be seen that both the scaling factor (Figure $7(\mathrm{~b})$ ) and the integral constants (Figure 7(c)) experienced the obvious variations with the magnified deformation phase. In Figure 7(b), the estimated scaling factor presents the shape of radial lines for the varied deformation phase. In Figure 7(c), the integral constants at each range pixels keep increasing or decreasing with the varied deformation phase. Meanwhile, it is observed that the scaling factor and integral constants become more and more distant from their true values when the deformation phase becomes larger and larger. Therefore, the standard deviations of the estimated IPS become larger and larger, as shown in Figure $7(\mathrm{~d})$. From this simulation, it is observed that the severe deformation signals in the interferogram can affect the estimation of the scaling factor and integral constants and consequently distort the IPS estimation by the azimuth offset method. Therefore, it should mask out the deformation signals when using the azimuth offsets method to correct the ionospheric error on SAR interferometry.

4.2. Real SAR Data. An ionospheric phase error was corrected by subtracting IPS (Figure $5(\mathrm{~d})$ ) from the original interferometric phase (Figure 4(b)). Then, two polynomial 


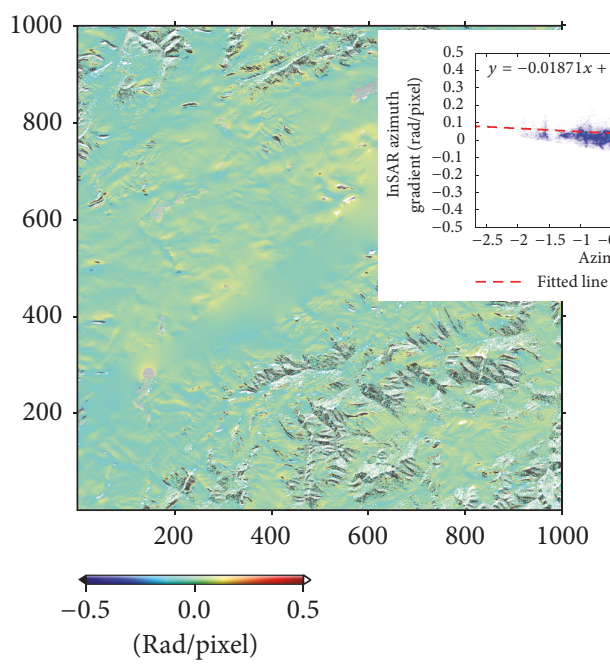

(a)

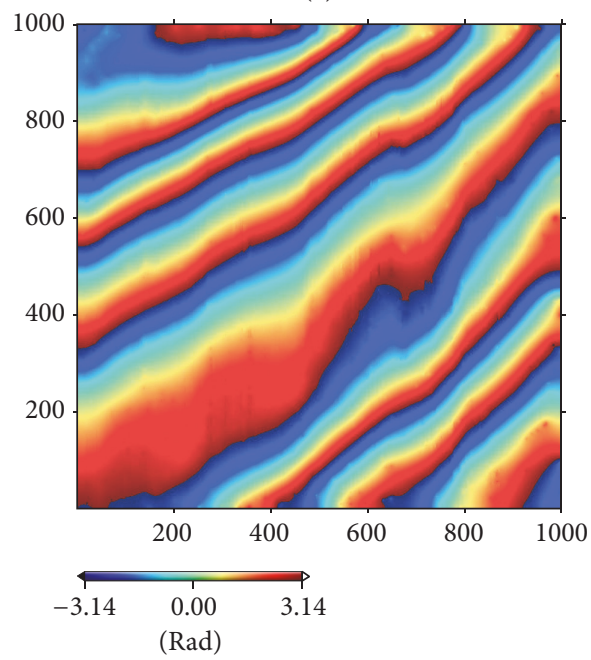

(d) (e)

Figure 5: (a) Azimuth gradient of interferometric phase for pair 2 in Table 1; (b) ionosphere-induced azimuth offset; (c) scattergram of (a) and (b), where the dashed red line represents the regressed line; (d) estimated IPS and corresponding VTEC (e).

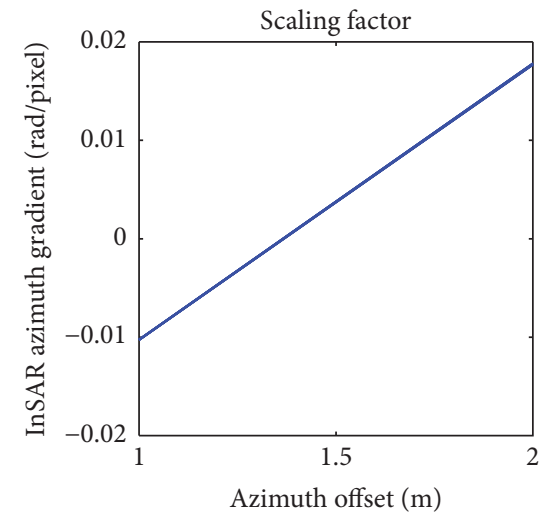

(a)

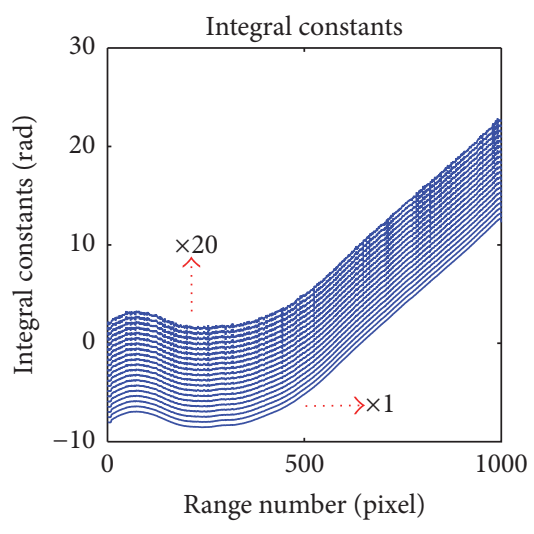

(b)

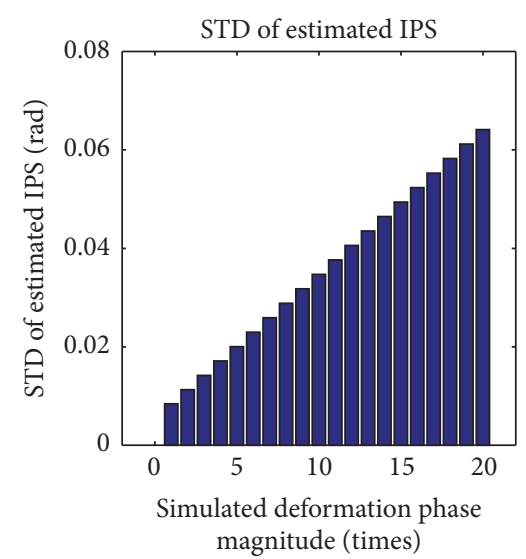

(c)

FIGURE 6: The variations of the scaling factor (a), integral constants (b), and standard deviation of the bias of estimated IPS (c) when the random noises were magnified from 1 to 20 times. 


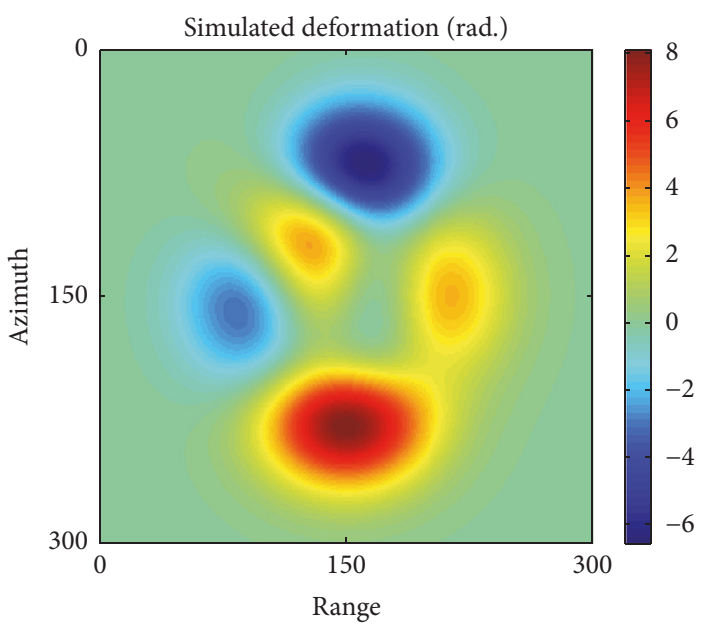

(a)

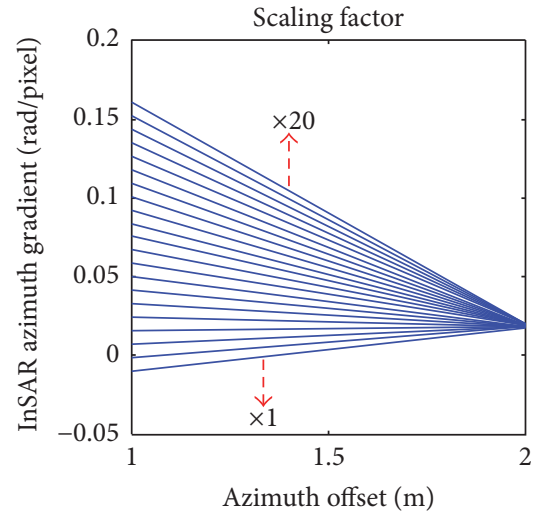

(b)

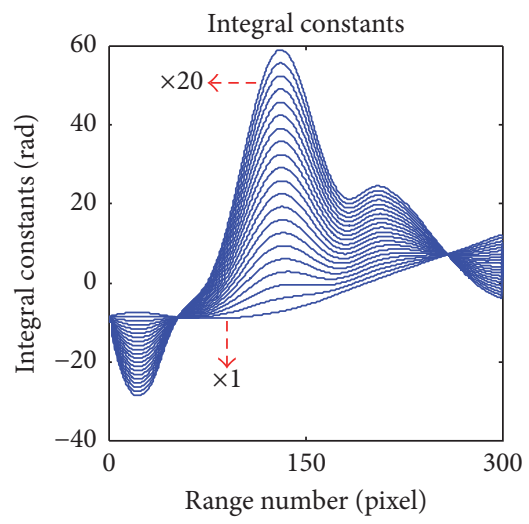

(c)

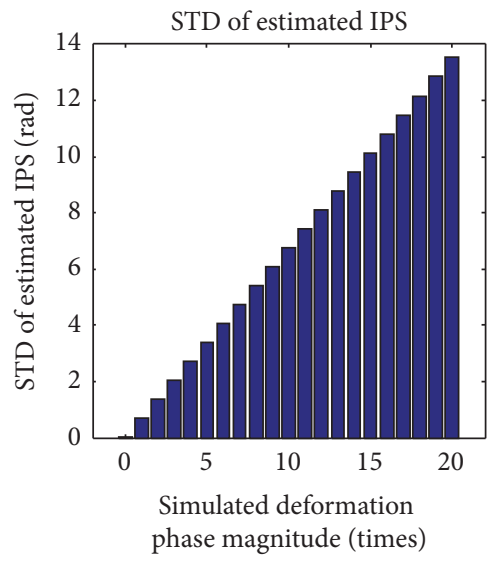

(d)

FIGURE 7: Simulated influence of deformation phase on the estimation of IPS. (a). Simulated deformation phase. The variations of the scaling factor (b), integral constants (c), and standard deviation of the bias of estimated IPS (d) with the magnified deformation phase from 1 to 20 times.

models were fitted to remove the residual orbital phase and atmospheric phase with respect to height, respectively. Lastly, the ionosphere- and orbit-corrected phase is shown in Figure 8(c). It is observed from Figure 8(c) that most longwavelength phase signals in Figure 4(b) have been corrected. However, the residual long-wavelength signal still remains, particularly at the upper-left and lower-right corners of the image. We think they were the residual orbital error and ionospheric phase noise.

Figure 8(a) shows the unwrapped phase of interferometric pair 1 in Table 1 . It was found that it was dominated by the coseismic deformation, meaning that it can be safely used to evaluate our ionospheric correction. Figure 8(d) displays the phase difference between Figures $8(\mathrm{a})$ and $8(\mathrm{~b})$ and can be simply regarded as the phase errors due to the ionospheric disturbance and inaccurate orbit. The mean and standard deviation values in Figure 8(d) are, respectively, -12.9 rad. and $5.3 \mathrm{rad}$. As for the ionosphere- and orbit-corrected image, the mean and standard deviation of phase errors in
Figure 8(e) are $6.5 \mathrm{rad}$. and $2.6 \mathrm{rad}$., respectively. Comparison of the statistics suggests that the absolute mean and standard deviation of phase difference after correction decreased by almost 2 times, compared to those before correction, as shown in Table 2 . This result validates the correction of the ionospheric error and orbital error by azimuth offset method. In order to further compare the coseismic deformation before and after correction, the phase values along profile $A A^{\prime}$ in Figures $8(\mathrm{a})-8$ (c) were extracted, as shown in Figure $8(\mathrm{f})$. It is noticed that the corrected phase (green line) is closer to the true coseismic deformation phase (blue line) than the original phase (red line) in the upper part of the earthquake surface rupture, demonstrating the efficiency of our correction. However, in the lower part, the correction of coseismic deformation phase is not significant when compared to the original phase. Moreover, further observation shows us that the corrected deformation phase was always above the true value. We think that this phenomenon was caused by the improper integral constant, which makes us underestimate the IPS. 


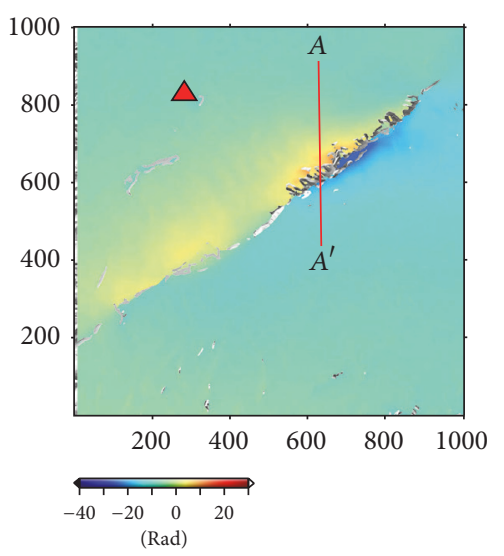

(a)

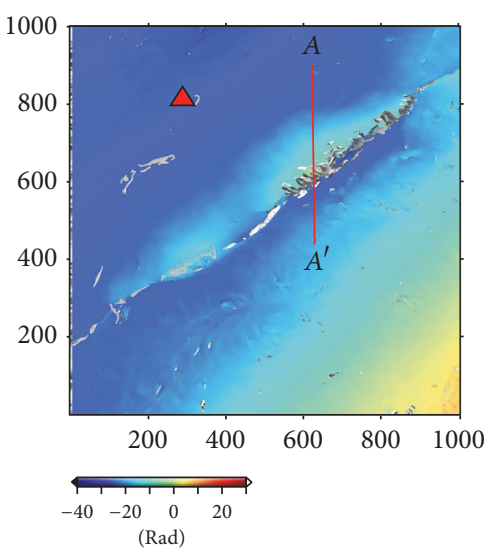

(b)

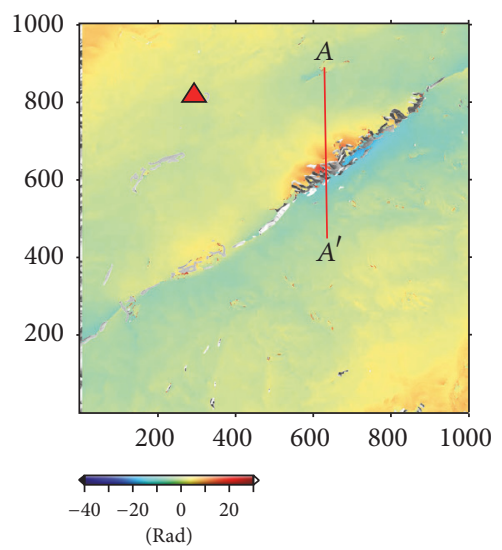

(c)

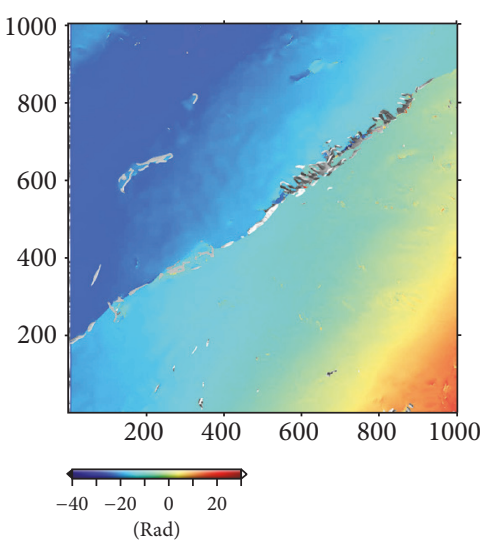

(d)

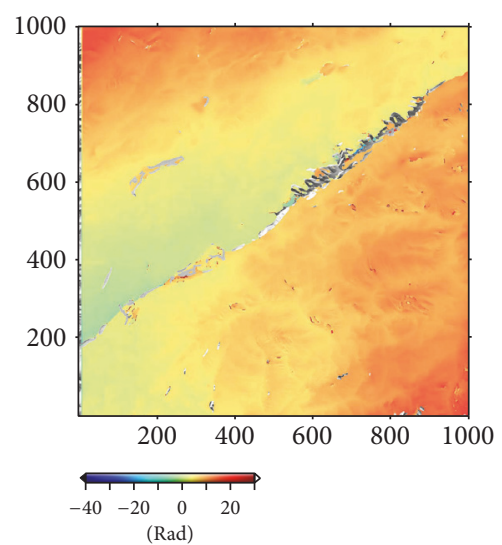

(e)

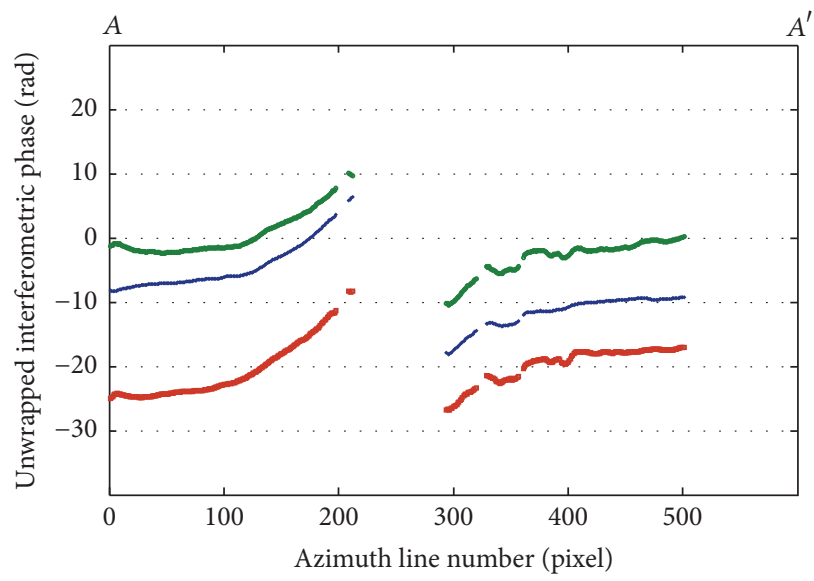

— $20100115-20100417$
-* 20100115-20100718
… Corrected 20100115-20100718

(f)

Figure 8: (a) Unwrapped phase for interferometric pair 1 in Table 1, where the red triangle shows the reference point; (b) unwrapped phase for interferometric pair 2; (c) ionosphere-corrected unwrapped phase for interferometric pair 2; (d) phase difference between (a) and (b); (e) phase difference between (a) and (c); (f) the phase values in the profile $A-A^{\prime}$ of (a)-(c). 
TABLE 2: The mean and standard deviation values of interferometric phase before and after ionospheric correction for Yushu coseismic interferogram.

\begin{tabular}{lcccc}
\hline Number & Interferogram & Mean (rad.) & Std (rad.) & Improvement rate \\
\hline$(1)$ & Original for pair 1 & 6.6 & 1.5 & \\
$(2)$ & Original for pair 2 & -12.9 & 5.3 & $51 \%$ \\
$(3)$ & Ionosphere-corrected for pair 2 & 6.5 & 2.6 & \\
\hline
\end{tabular}

\section{Discussion and Conclusion}

Ionospheric effect has limited the further development of InSAR technique. To mitigate its effect, azimuth offset method was developed based on the approximate linear relationship between the azimuth gradient of IPS and the azimuth pixel shift. However, the performance of this method has not been fully investigated. In this situation, the study made a performance analysis of azimuth offset methods in mitigating the ionospheric phase delay error on SAR interferometry through processing the simulated and real SAR data. Based on this study, we may draw the following conclusions.

(1) Azimuth offset method can effectively mitigate the ionospheric phase delay error for SAR interferometry. The result of real SAR data regarding the Yushu earthquake shows that the mean and standard deviation of phase error after correction by using azimuth offset method decreased by almost 2 times compared to those before correction. Meanwhile, the corrected coseismic deformation phase was closer to the true value than the uncorrected phase in the upper part of the earthquake surface rupture. Both results prove the efficiency of this method.

(2) The random noise in the interferogram can induce the improper estimation of integral constants when using azimuth offset method to mitigate the ionospheric phase delay error. The simulated experiment shows that the scaling factor is not obviously affected by the magnified random noise. However, the integral constants experience an obvious variation with the magnified random noise. This indicates that random noise may lead to the estimation error of integral constants and consequently produce the discontinuous pinstripe along the range direction in the estimated IPS map.

(3) The severe deformation signals in the interferogram can affect the estimation of scaling factor and integral constants when using azimuth offset method to mitigate the ionospheric phase delay error. The simulated experiment shows that both scaling factor and integral constants become more and more distant from their true values when the deformation phase becomes larger and larger, which may distort the IPS estimation by the azimuth offset method. Therefore, it should mask out the deformation signals when using the azimuth offsets method to correct the ionospheric error on SAR interferometry.

\section{Conflicts of Interest}

The authors declare that they have no conflicts of interest.

\section{Acknowledgments}

This research is funded by Chang'an University (Xian, China) through Natural Science Foundation of China (NSFC) Projects (nos. 41504005 and 41674001), the Fundamental Research Funds for the Central Universities (no. 310826171005), Natural Science Basic Research Plan in Shaanxi Province of China (2016JM4005), and National Program on Key Basic Research Project (973 Program) (no. 2014CB744703) and by Hong Kong Polytechnic University (Hong Kong, China) through Natural Science Foundation of China (NSFC) Projects (no. 41374013).

\section{References}

[1] D. Massonnet, M. Rossi, C. Carmona et al., "The displacement field of the Landers earthquake mapped by radar interferometry," Nature, vol. 364, no. 6433, pp. 138-142, 1993.

[2] C. Wang, X. Ding, X. Shan, L. Zhang, and M. Jiang, "Slip distribution of the 2011 Tohoku earthquake derived from joint inversion of GPS, InSAR and seafloor GPS/acoustic measurements," Journal of Asian Earth Sciences, vol. 40, no. 4, pp. 935942, 2012.

[3] P. A. Rosen, S. Hensley, H. A. Zebker, F. H. Webb, and E. J. Fielding, "Surface deformation and coherence measurements of Kilauea Volcano, Hawaii, from SIR-C radar interferometry," Journal of Geophysical Research E: Planets, vol. 101, no. 10, pp. 23109-23125, 1996.

[4] C. Zhao, Z. Lu, Q. Zhang, and J. de la Fuente, "Large-area landslide detection and monitoring with ALOS/PALSAR imagery data over Northern California and Southern Oregon, USA," Remote Sensing of Environment, vol. 124, pp. 348-359, 2012.

[5] L. Zhang, Z. Lu, X. Ding, H.-S. Jung, G. Feng, and C.-W. Lee, "Mapping ground surface deformation using temporarily coherent point SAR interferometry: application to Los Angeles Basin," Remote Sensing of Environment, vol. 117, pp. 429-439, 2012.

[6] C. Wicks Jr., W. Thatcher, and D. Dzurisin, "Migration of fluids beneath yellowstone caldera inferred from satellite radar interferometry," Science, vol. 282, no. 5388, pp. 458-462, 1998.

[7] P. Mateus, G. Nico, R. Tome, J. Catalão, and P. M. A. Miranda, "Experimental study on the atmospheric delay based on GPS, SAR interferometry, and numerical weather model data," IEEE Transactions on Geoscience and Remote Sensing, vol. 51, no. 1, pp. 6-11, 2013.

[8] R. Hanssen, Radar Interferometry: Data Interpretation and Error Analysis, Kluwer Academic, Boston, Massachusetts, Mass, USA, 2001.

[9] S. H. Bickel and R. H. T. Bates, "Effects of magneto-ionic propagation on the polarization scattering matrix," Proceedings of the IEEE, vol. 53, no. 8, pp. 1089-1091, 1965. 
[10] F. J. Meyer and J. B. Nicoll, "Prediction, detection, and correction of faraday rotation in full-polarimetric L-band SAR data," IEEE Transactions on Geoscience and Remote Sensing, vol. 46, no. 10, pp. 3076-3086, 2008.

[11] A. L. Gray, K. E. Mattar, and G. Sofko, "Influence of ionospheric electron density fluctuations on satellite radar interferometry," Geophysical Research Letters, vol. 27, no. 10, pp. 1451-1454, 2000.

[12] U. Wegmüller, C. Werner, T. Strozzi, and A. Wiesmann, "Ionospheric electron concentration effects on SAR and INSAR," in Proceedings of the 2006 IEEE International Geoscience and Remote Sensing Symposium, IGARSS, pp. 3731-3734, August 2006.

[13] J. Chen and H. A. Zebker, "Ionospheric artifacts in simultaneous L-band InSAR and GPS observations," IEEE Transactions on Geoscience and Remote Sensing, vol. 50, no. 4, pp. 1227-1239, 2012.

[14] K. E. Mattar and A. L. Gray, "Reducing ionospheric electron density errors in satellite radar interferometry applications," Canadian Journal of Remote Sensing, vol. 28, no. 4, pp. 593-600, 2002.

[15] D. Raucoules and M. De Michele, "Assessing ionospheric influence on L-Band SAR data: Implications on coseismic displacement measurements of the 2008 Sichuan Earthquake," IEEE Geoscience and Remote Sensing Letters, vol. 7, no. 2, pp. 286-290, 2010.

[16] H.-S. Jung, D.-T. Lee, Z. Lu, and J.-S. Won, "Ionospheric correction of SAR interferograms by multiple-aperture interferometry," IEEE Transactions on Geoscience and Remote Sensing, vol. 51, no. 5, pp. 3191-3199, 2013.

[17] H.-S. Jung and W.-J. Lee, "An improvement of ionospheric phase correction by multiple-aperture interferometry," IEEE Transactions on Geoscience and Remote Sensing, vol. 53, no. 9, pp. 4952-4960, 2015.

[18] Z. Liu, H.-S. Jung, and Z. Lu, "Joint correction of ionosphere noise and orbital error in L-Band SAR interferometry of interseismic deformation in southern California," IEEE Transactions on Geoscience and Remote Sensing, vol. 52, no. 6, pp. 3421-3427, 2014.

[19] F. Meyer, R. Bamler, N. Jakowski, and T. Fritz, “The potential of low-frequency SAR systems for mapping ionospheric TEC distributions," IEEE Geoscience and Remote Sensing Letters, vol. 3, no. 4, pp. 560-564, 2006.

[20] B. M. Kampes, Radar Interferometry: Persistent Scatterer Technique, Springer-Verlag, Dordrecht, Netherlands, 2006.

[21] N. B. D. Bechor and H. A. Zebker, "Measuring two-dimensional movements using a single InSAR pair," Geophysical Research Letters, vol. 33, Article ID L16311, pp. 1-5, 2006. 


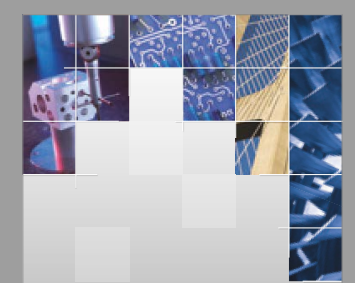

\section{Enfincering}
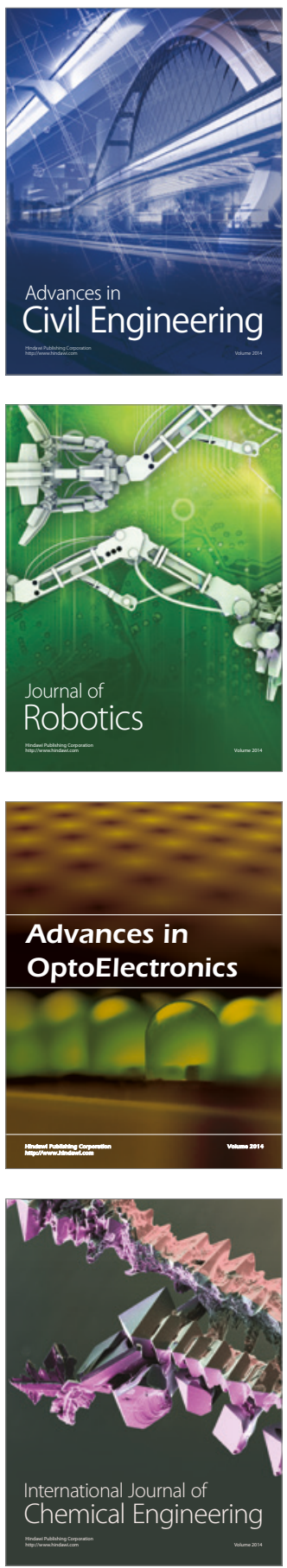

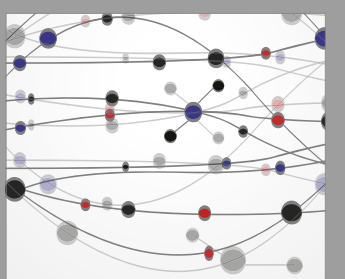

The Scientific World Journal

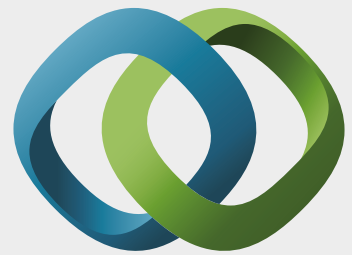

\section{Hindawi}

Submit your manuscripts at

https://www.hindawi.com
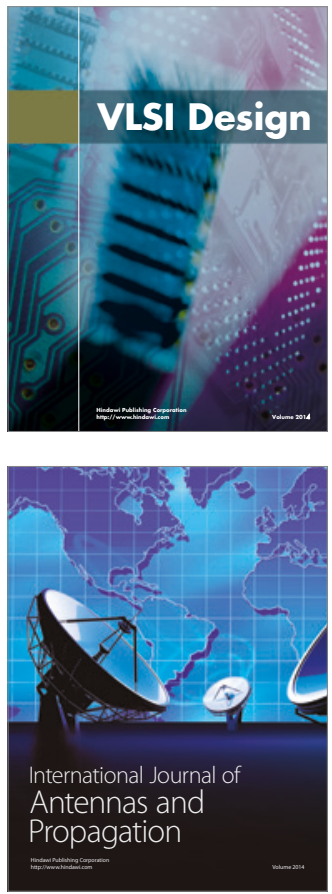

\section{Rotating}

Machinery
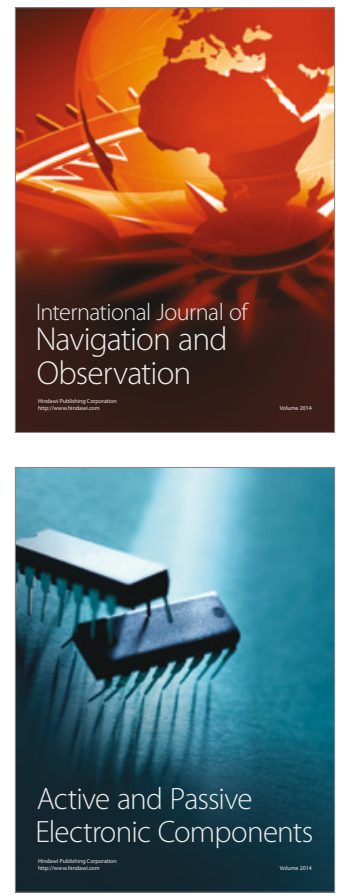
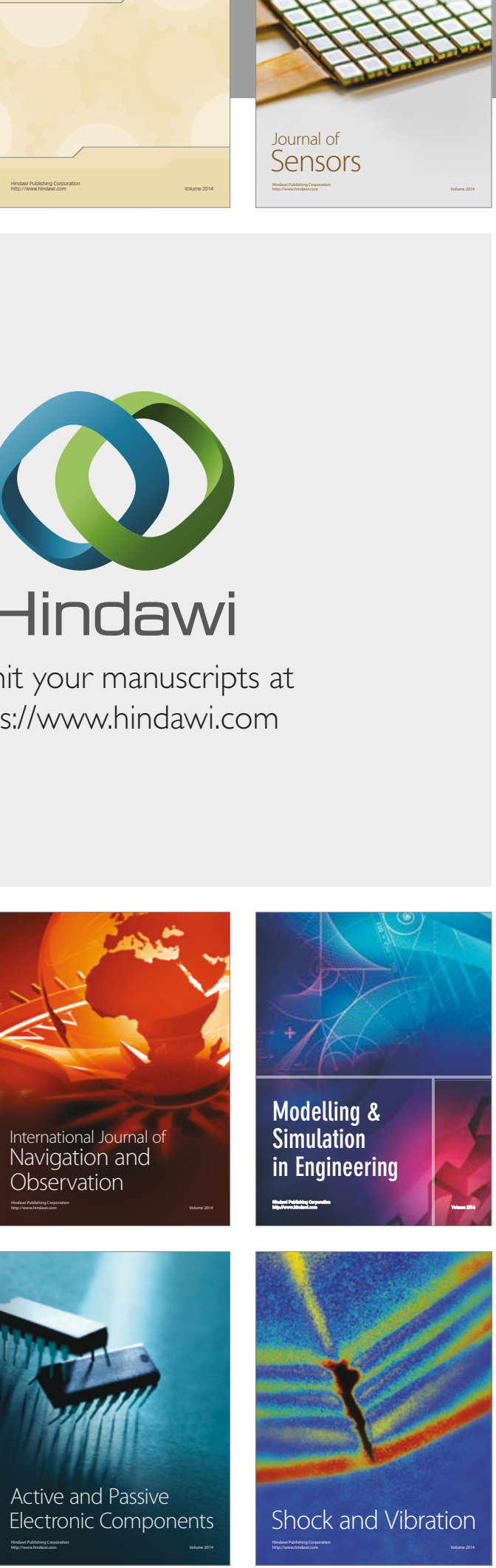
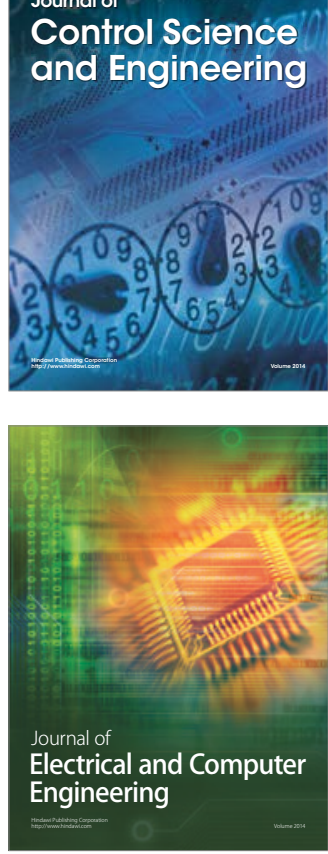

Distributed

Journal of

Control Science

and Engineering
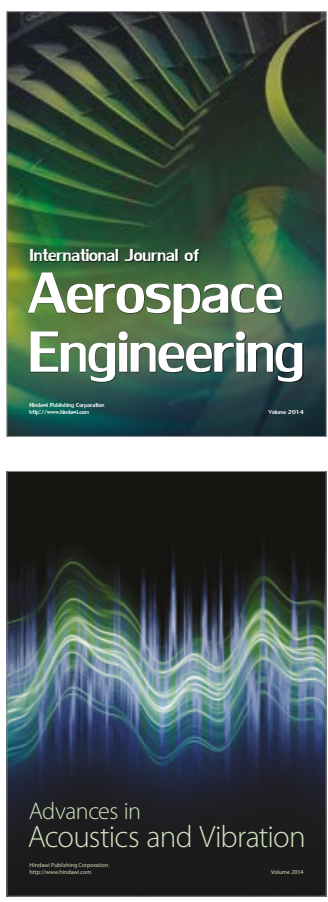

Sensor Networks 